\title{
Comparison of Multiple Object Tracking Performance Between Professional and Amateur eSport Players as Well as Traditional Sportsmen
}

\author{
Konstantin Wechsler, German Sport University Cologne, Germany \\ Peter Bickmann, German Sport University Cologne, Germany \\ Kevin Rudolf, German Sport University Cologne, Germany \\ (iD) https://orcid.org/0000-0001-9815-3475 \\ Chuck Tholl, German Sport University Cologne, Germany \\ Ingo Froböse, German Sport University Cologne, Germany \\ Christopher Grieben, German Sport University Cologne, Germany
}

\begin{abstract}
High performance in multiple object tracking paradigms is associated with well-trained visuo-spatial abilities, visual memory, and divided attention. These abilities are essential for both traditional sport and eSport. The present study compared the tracking performance of professional as well as amateur eSport players and traditional sportsmen. Professional eSport players outperformed amateurs, while no other group differences were found. Positive association of eSport playtime and tracking performance as well as elevated tracking scores of the entire study cohort compared to normal population indicate a connection of eSport and sport activity and tracking performance.
\end{abstract}

\section{KEYWORDS}

eSport, Gaming, Perceptual-Cognitive Abilities, Performance, Playtime, Professional Players, Visual Tracking

\section{INTRODUCTION}

Electronic Sport (esport) is a widespread and increasingly growing economic, cultural, and scientific phenomenon. With an audience up to 495 million (Newzoo, 2020) and an expanding player base of both casual and professional players, the competition for immense prize pools on the top level is getting more and more intense. The competition in esports takes place on different levels. Amateurs on the lowest level compete in tournaments for fun and without any monetary motivation, while professional players earn a relevant amount of income by playing, either alone or in teams. The main distinction between amateurs and professionals in esport is not clearly or officially defined, mostly it is based on the presence of relevant income (Lipovaya et al., 2018) earned by competition in tournaments. This classification is applied identically in many traditional sports, such as soccer or tennis, where players are defined as professionals if they are above a certain income limit or play in a certain

\section{DOI: 10.4018/IJER.20210101.oa2}

This article published as an Open Access article distributed under the terms of the Creative Commons Attribution License (http://creativecommons.org/licenses/by/4.0/) which permits unrestricted use, distribution, and production in any medium, provided the author of the original work and original publication source are properly credited. 
division. The practice of sport (or esport) of professionals is therefore their profession. The current route to excellence in esport is still unclear (Pedraza-Ramirez et al., 2020), there are no developmental stages defined and no real career plans are established (Abbot \& Collins, 2004). Currently only some thousand players can be accounted as professional in the esport context (Nielsen \& Karhulahti 2017). Players compete in diverse fields including, real time strategy games, e.g., StarCraft II (Blizzard Entertainment, 2010). or Age of Empires III (Ensemble Studious, 2005), multiplayer online battle arena games, e.g., Defence of the Ancients 2 (Valve Corporation, 2013) or League of Legends (Riot Games, 2009) or first-person shooters, e.g., Call of Duty: Modern Warfare (Infinity Ward, 2019) or Counter-Strike: Global Offensive (Valve Corporation, 2012). Other competitive games and simulations are based on traditional sports such as soccer, e.g., FIFA $19=$ Fédération Internationale de Football Association (EA Vancouver, 2018) or PES $20=$ Pro Evolution Soccer (PES Productions, 2019) or racing, e.g., Gran Turismo Sport (Polyphony Digital, 2017) or Forza Motorsport 4 (Turn 10 Studios, 2011) (Bányai et al., 2019; Taylor, 2011).

All kinds of esport games require the player to react as fast as possible to versatile, primarily visual stimuli, to make quick as well as correct decisions in complex situations, and to inhibit unnecessary information along the way (Kowal et al., 2018). This is in line with the thoughts of Happonen and Minashkina (2019) as well as Himmelstein et al. (2017), who propose that quick decision-making, along with other mental factors, might be important to achieve victory. Accurate perception and tracking of movement are fundamental. For instance, players need to track their own moving avatar in League of Legends while simultaneously paying attention to the changing positions of multiple enemies and allies. Another example is the football simulation FIFA. Similar to real-world football, players need to keep focus on the ball-carrying avatar, opponents, and possible support avatars of their own team. This is particularly difficult, because all of these related possible focus points are in motion. This puts high demands on perceptual processing and central and peripheral attentional skills (Bavelier et al., 2011; Torner et al., 2019).

An essential ability to succeed in those situations has been given the name "multiple object tracking" (MOT) in the literature. MOT has been the subject of research for more than 30 years, first addressed by Pylyshyn and Storm (1988). Subjects in their experiment were able to track up to 4-5 moving objects in a confined space, bouncing off each other and the borders. The authors deduced that participants could not only use eye movements to follow the objects, but also an attentional locus that is independent of those movements. As a result of this finding, several models (grouping, attention switching, multifocal attention, preattentive indexes and object files) were proposed in order to explain the mechanisms of MOT in humans. Cavanagh \& Alvarez (2005) as well as Oksama \& Hyönä (2004) described these models in detail: The grouping and switching approaches only require a single focus of attention. The moving objects are grouped into a higher order object and the participant can then track the changing shape and handle the task with only one attentional channel. Additionally, when targets share common motion (e.g. move in the same direction), they group more strongly and tracking becomes easier. Switching required the participants to cycle through the targets and keeping track of all positions and anticipated locations. The multifocal attentional approach assumes that each target requires an independent focus of attention and this focus needs to be held until the movement of objects stops. The preattentive indexes model is somewhat similar to the multifocal model, it differs in the view that there is no explicit attentional focus needed to follow an object. The object files model is not an alternative model to e.g. multifocal attention, instead it describes in more detail which constituent processes are required to accumulate information about each moving object.

Summing up, MOT addresses the question, how well participants can perform in visuo-spatial, visual memory, multifocal and divided attentional tasks. Those tasks are all crucial for esport- as well as traditional sport performance. 


\section{BACKGROUND}

As mentioned, there is a clear link between MOT and esport performance. For example, the differentiation of performance has been subject of esport science, as regular gamers outperformed novices in different attentional tasks (Hubert-Wallander et al., 2011) and gaming experts could track objects at greater speed compared to non-gamers (Boot et al., 2008). There are two feasible explanations for these differences in performance. One is given by multiple studies which have shown that playing video games can have positive effects on attentional and visuo-spatial cognition such as MOT (Bejianki et al., 2014; Ding et al., 2018; Trisolini et al., 2018). These findings indicate that playing video games regularly improves those aspects of cognition and therefore the ability of MOT. Another possible explanation for the differences between gaming experts and novices is, that people with a better initial ability of MOT might be more successful in esport competitions and therefore engage more regularly and tend to be more successful.

MOT ability is also relevant in traditional team ball sports which constantly challenge players with complex and dynamic motions (Cavanagh et al., 2014; Memmert, 2009; Qiu et al., 2018; Scholl, 2009; Williams et al., 2006). The ability plays an important role in individual sports such as karate or fencing, where athletes need to distinguish and simultaneously process the cues of the opposing body during a sequence of attacks or counterattacks (Mouton \& Oberle, 2007). The assessment of MOT helped to differentiate elite athletes from novice and non-athlete populations (Alves et al., 2013; Faubert, 2013; Faubert \& Sidebottom 2012; Qiu et al., 2018). Voss et al. (2009) also found in their review that expert athletes performed better in tasks that required processing speed and visual attention compared to amateurs. MOT paradigms have not only been used for performance assessment, but also to successfully train soccer players' decision making (Romeas et al., 2016) and other sporting related abilities (Junye et al., 2015). Fleddermann et al. (2019) also found better training effects of a MOT training on perceptual-cognitive tasks in comparison to traditional training. Moreover, MOT correlated to the performance of players in the National Basketball Association of the United States of America (Mangine et al., 2014). Summing up, MOT is seen to be relevant in both esport and traditional sport. It has, among other goals, been used to differentiate performance in both fields. Up to now, studies have compared either experts and novices in one of the two fields or, in the case of esport, collated gamers to a non-gamer population. No comparison between differently skilled advanced esport players and sportsmen previously has been made (García-Lanzo et al., 2018) and studies that involve esport players from the highest level are lacking, possibly because of the low accessibility of professional players.

\section{AIM OF THE STUDY AND HYPOTHESES}

The current study is an experimental and practically oriented approach to address this gap by comparing the MOT ability of three relevant groups of participants: professional and amateur esport players, as well as traditional sportsmen. The traditional sportsmen implemented in this study might also function as a control group for the other two, because they did not play esport games regularly. The following three hypotheses are formulated:

- Professional esport players have superior attentional and visuo-spatial cognition compared to novice esport players, possibly as a result of their greater practice time in esport games.

- Professional esport players and traditional sportsmen show comparable attentional and visuospatial cognition, possibly as a result of their practice times in either traditional sport or esport

- Traditional sportsmen have superior attentional and visuo-spatial cognition compared to novice esport players, possibly as a result of their practice time in traditional sport 
Table 1. Descriptive data for professional and amateur esport players

\begin{tabular}{|l|l|l|l|}
\hline Player Group & Age (years) & Esport experience (years) & Game time (h) / Week \\
\hline $\begin{array}{l}\text { Professional esport players ( } \mathrm{n} \\
=19,1 \text { female) }\end{array}$ & $23.00 \pm 3.11$ & $12.58 \pm 4.26$ & $35.95 \pm 13.67$ \\
\hline $\begin{array}{l}\text { Amateur esport players } \\
(\mathrm{n}=22,2 \text { females) }\end{array}$ & $24.91 \pm 6.19$ & $11.30 \pm 6.00$ & $22.14 \pm 12.78$ \\
\hline
\end{tabular}

\section{METHODS}

\section{Study Design and Participants}

The current cross-sectional study was conducted between October 2018 and April 2019. Young adults were recruited via notices in public places or via the websites of the German Sport University Cologne and the research project. Respondents were accepted for the study if they met the following inclusion criteria, as assessed by self-report: (1) they were physically and mentally healthy, including normal or corrected to normal vision and with no color blindness, and (2) they did not suffer from blind spots in their view. Furthermore, three definitions were used to classify the participants: Professional players earned money as a main income by playing competitively in tournaments. Amateur players were playing competitively in tournaments but did not earn money as a main income by doing so. Traditional sportsmen did not play video games more than five times a month and performed their main sport at least twice a week. None of them earned money as a main income by performing their sport.

All self-reports were done via self-designed questionnaires, in accordance with a physician. In addition to the self-report, blind spot diagnosis was carried out with the "multifixation campimeter (basic)" software (St Paul's Eye Unit; Royal Liverpool University Hospital) and the "EyesCream II" software (MGM). Both software packages are publicly available and were calibrated and used according to the developers' specifications.

Table 1 represents the descriptive data for the professional and amateur esports players. The data for game time per week refer to the last six weeks. The games played by professional players were FIFA $20(\mathrm{n}=13)$, League of Legends $(\mathrm{n}=4)$, PlayerUnknown's Battlegrounds (PUBG Corporation, 2017, $\mathrm{n}=1$ ) and. Trackmania 2: Stadium (Nadeo,2013, n=1). Amateur player's main games were League of Legends $(\mathrm{n}=10)$, FIFA $(\mathrm{n}=5)$, Counter-Strike: Global Offensive $(\mathrm{n}=3)$, PlayerUnknown's Battlegrounds $(\mathrm{n}=2)$, Clash Royale (Supercell, 2016. $\mathrm{n}=1$ ) and Madden NFL $2019=$ National Football League (EA Tiburon, 2019, $\mathrm{n}=1$ ). Amateur players often played more than one game.

Table 2 represents the descriptive data for the traditional sportsmen. The data for game time and traditional sports per week refer to the last six weeks. Main sports performed were team sports (9; e.g., basketball, volleyball, soccer, handball), track and field (5) and fitness (4) among others and in combination.

The overall sample size of our study was 59 (19 professional players, 22 amateur players and 18 traditional sportsmen/control group) and is therefore higher than the larger sample sizes in the literature. For example:

- Bejianki et al. (2014) compared 10 to 11 participants in training a study

Table 2. Descriptive data for traditional sportsmen

\begin{tabular}{|l|l|l|l|}
\hline & Age (years) & Sports experience (years) & Sports time (h) / Week \\
\hline $\begin{array}{l}\text { Traditional sportsmen }(\mathrm{n}= \\
18,11 \text { females) }\end{array}$ & $23.72 \pm 3.41$ & $12.22 \pm 4.60$ & $8.40 \pm 4.60$ \\
\hline
\end{tabular}


Table 3. Number of groups and overall sample size of the present and earlier studies

\begin{tabular}{|l|l|l|}
\hline Study & Number of groups & Overall sample size \\
\hline Present study & 3 & 59 \\
\hline Bejianki et al. (2014) & 2 & 21 \\
\hline Boot et al. (2008) & 2 & 21 \\
\hline Hubert-Wallander et al. (2011) & 2 & 21 \\
\hline Ding et al. (2018) & 3 & 40 \\
\hline Trisolini et al. (2018) & 2 & 45 \\
\hline
\end{tabular}

- $\quad$ Boot et al. (2008) compared 11 experts to 10 novices

- Hubert-Wallander et al. (2011) compared 10 videogamers to 11 non-gamers

- Ding et al (2018) compared 10 professional players, 10 semi-professional players and 20 control-participants

- Trisolini et al. (2018) compared 30 videogamers to 15 non-gamers

Table 3 shows the number of groups and overall sample size of comparable studies.

The study was approved by the Ethics Commission of the German Sport University Cologne (reference: 053/2018), and all participants signed an informed consent statement in accordance with the Declaration of Helsinki. One participant was underage (17 years old) and presented a legal guardian's consent to be able to participate.

\section{Multiple Object Tracking Testing}

Testing software was developed by Faubert and Sidebottom (2012) and is commercially available (Neurotracker@, CogniSens Athletic, Inc., Montreal, Quebec, Canada). All Participants completed three sessions, each consisting of 20 trials. The software functioned as follows: In each trial, a transparent cube with a green cross in the centre was presented. Inside the cube, eight identical yellow balls were randomly distributed. Four of the balls were highlighted for two seconds. After returning to original colour, the balls moved individually throughout the entire cube for eight seconds. The movements were affected by collisions with the walls of the cube or other balls. At the end of the eight-second period, all balls stopped and the camera moved very slowly around the cube to avoid possible occlusion of subjacent balls. Each ball was assigned a number from 1 to 8 by the software and participants were instructed to identify the four balls originally highlighted at the beginning of the trial (for whole sequence see Figures 1,2 and 3).

The speed of the starting trial of each session was identical for every participant, but it was different in session one compared to session two and three. Session one started at speed 0.3, whereas session two and three started at the speed of 0.7. The speed at which the balls moved in every following trial was dependent on the correct identification of the highlighted balls and was adjusted immediately. Speed adaptation followed a one up one down staircase fashion (Levitt, 1971) in which multiple correct trials in succession lead to a heightened increase for the third and the subsequent correct trials. All these specifications are used as standard configuration by the software and are recommended by the developers.

In the first session, participants worked on the 2D version of the task; for sessions two and three, the $3 \mathrm{D}$ version was used. The balls were then displayed as two overlapping $2 \mathrm{D}$ images with slightly different perspectives, as can be seen in Figure 4. 
Figure 1. Highlighting of the relevant balls

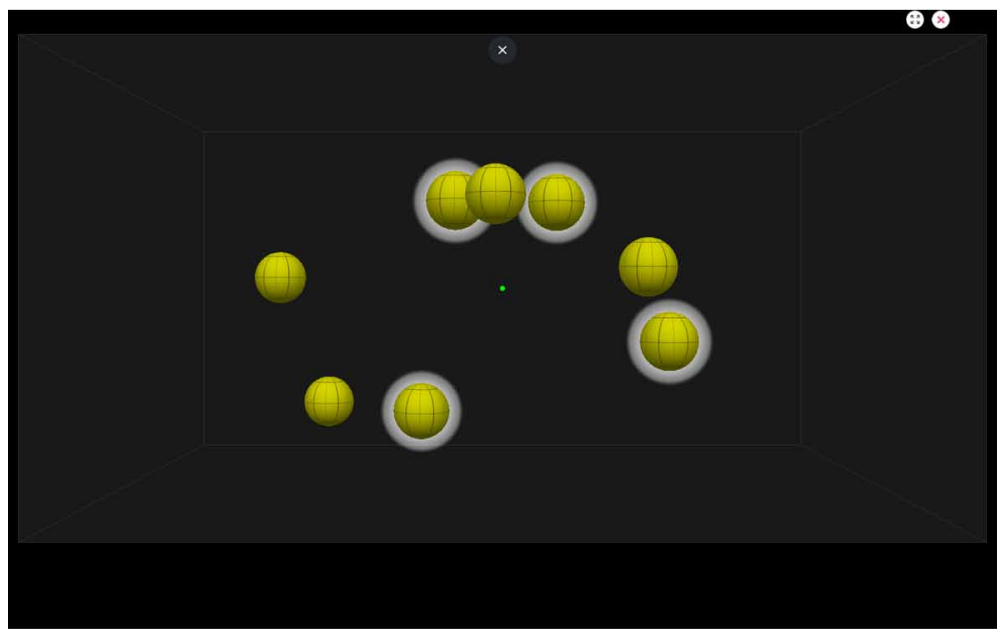

When perceived with stereoscopic glasses, these two images converted into a 3D image. This is again based on developer's recommendations. None of the participants reported problems with either of the two versions.

\section{General Procedures}

Testing was conducted by experienced instructors that were specially trained in explaining, executing and monitoring the tests. Training of instructors was conducted in consultation with the developers of the software and took place in the laboratory where later testing was executed. It included details about the setup of the testing computer as well as all details described in the previous section. The testing procedure itself was part of an extensive test battery that was carried out within the same day, which is why only a limited time slot could be granted for the MOT test covered in this study.

Figure 2. All balls in movement

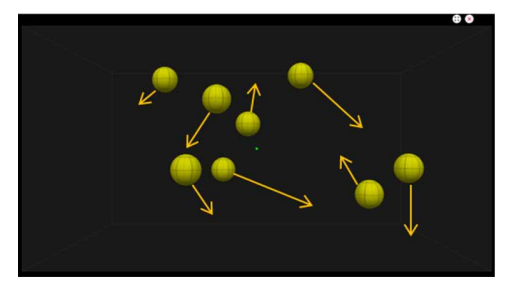

Figure 3. Balls with assigned numbers

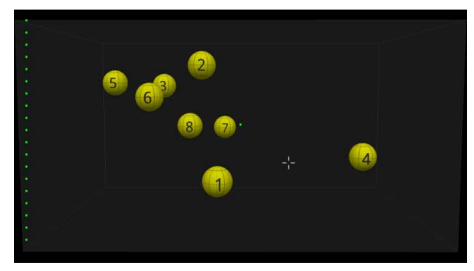




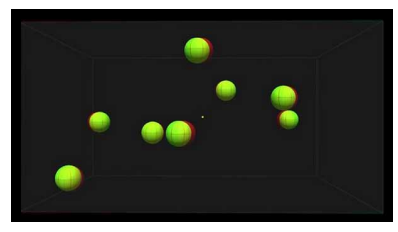

The feasible three sessions of MOT testing took 20 to 25 minutes, dependent on voluntary and freely selectable breaks in between sessions.

Participants were tested separately in the laboratory and were sitting in front of a PC in an otherwise darkened room and used either the mouse or keypad to identify the highlighted balls after a trial. Participants could freely choose their individual optimal distance from the screen and adjust keypad and mouse placement or mouse speed or if needed. No calibration of the software or hardware was necessary. First, the examiner read aloud the instructions and explained the task, which included a practice trial that could be repeated if necessary. Instructions included the procedure of the overall upcoming testing, the possibility to take a break in between sessions, the procedure of a single trial as well as the speed adaptation as a consequence of correct or false identification of the moving objects (see previous section).

He then withdrew from participants' view and supervised the procedure without disturbing or interacting with the participant. No retesting of any participant was necessary. Retesting should also be considered with caution, because possible training effects could influence the results.

\section{Data Analysis}

The score value of each session was used as the dependent variable for the study. The score was determined to be the fastest speed at which participants could correctly identify all four highlighted balls $50 \%$ of the time. The score was automatically calculated by the testing software.

Means of session one, two and three were separately calculated for each participant. Afterwards, means of session one, two and three were calculated for the three groups (professional players, all amateur players and all traditional sportsmen) separately. Because of software recording issues, a total of three sessions from three different participants were not recorded. Because of this missing data, $1.70 \%$ of those means could not be calculated and were instead imputed from other participants' means. No outliers were excluded from statistical analyses because all means were within the range $\mathrm{M} \pm$ 3.29 SD, a criterion adopted from Tabachnick and Fidell (2001), with M and SD representing the mean and standard deviation across trials and participants.

Because of the different starting values for the scores and 2D (session one) vs 3D versions of the task, session one had to be analysed separately from sessions two and three. A one-way analysis of variance (ANOVA) with the mean score of session one as dependent variable and the independent variable "group" (professional and amateur esport players as well as traditional sportsmen) was conducted. Additionally, a repeated-measures ANOVA with the factors session (two and three) and the between-subject factor group was conducted. The degrees of freedom were not adjusted since the assumption of sphericity was not violated in any ANOVA.

Two-sample t-tests were used to compare esport playtime of professional players and amateur players in hours per week and overall (years).

To examine possible associations between MOT score values and esport playtime (hours per week), overall esport participation (years), sport activity (hours per week) and overall sport participation (years), partial Pearson correlations were calculated for both esport player groups combined and the separate group of sportsmen. 


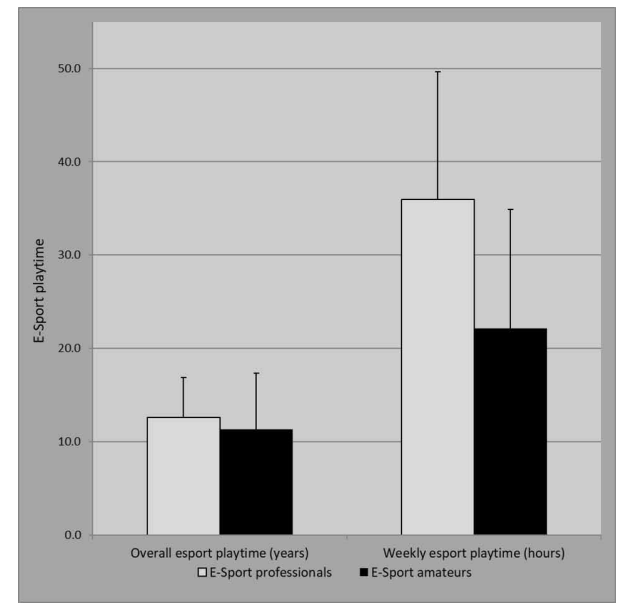

All calculations were controlled for age and gender, to prevent an influence of these factors, since there is an inevitable gender distribution in esport player groups. Because there were more female participants in the sportsmen group, all analysis were conducted including and excluding female participants, in case there were differences related to the gender. Results were tested for significant differences between male and female participants within the three groups, as well as for differences between the three groups including and excluding female participants. None of the analysis indicated that male and female participants differed in any outcome. Therefore, female participants were not excluded in statistical analysis to strengthen the power of the data through a larger number of participants. Additionally, we controlled all ANOVAS by using age and again gender as covariates and again found no significant differences. Correlation analysis were controlled by using partial Pearson correlations with control variables age and gender.

\section{RESULTS}

Experience and playtime data are presented in Table 1 and 2 in the study design and participant section. T-test showed that professional players played significantly more esport / week $(t(39)=-$ $3.342, p<0.05)$, as displayed in Figure 5. T-test did not show any difference in overall esport playtime (years). Figure 5 shows mean esport playtime + standard deviation overall (years) and over the last six months (weekly hours) of professional and amateur esport players.

As can be seen in Table 4, the one-way ANOVA revealed, that scores differed between groups in session one $\left(F(2,56)=3.380 ; p<0.05, \eta \mathrm{p}^{2}=0.108\right)$ Bonferroni post-hoc tests further differentiated that professional players had significantly $(p<0.05)$ higher scores compared to amateur players in session one $(0.300,95 \%$ - CI [0.01, 0.58]). Figure 6 accordingly shows mean score values + standard deviations of the three sessions across individuals in all three groups. Dotted horizontal lines indicate starting speed of sessions. The pervaded vertical line separates session one (2D) and session two/ three (3D).

The repeated-measures ANOVA for session two and three yielded a significant effect of session $\left(\mathrm{F}(2,56)=4.427 ; \mathrm{p}<0.05, \eta \mathrm{p}^{2}=0.073\right.$. Scores were significantly higher in session three compared to session two (post hoc: $\mathrm{p}<0.05 ; 0.088,95 \% \mathrm{CI}[0.04,0.172]$ ), but the interaction session*group was not significant, which means that no group differences were found between the two sessions.

Pearson correlations revealed positive associations between hours of esport / week over the last six months and the score values of session one $(r=0.421 ; p<0.05)$, as plotted in Figure 7 . 
Table 4. Mean score values \pm standard deviations of the three MOT sessions across individuals in the three groups

\begin{tabular}{|l|l|l|l|}
\hline Group & Session 1 & Session 2* & Session 3* \\
\hline $\begin{array}{l}\text { Professional esport players } \\
(\mathrm{n}=19)\end{array}$ & $1.25 \pm 0.34^{*}$ & $1.47 \pm 0.41$ & $1.56 \pm 0.54$ \\
\hline $\begin{array}{l}\text { Amateur esport players } \\
(\mathrm{n}=22)\end{array}$ & $0.95 \pm 0.39^{*}$ & $1.23 \pm 0.47$ & $1.35 \pm 0.51$ \\
\hline $\begin{array}{l}\text { Traditional sportsmen (n } \\
=18)\end{array}$ & $1.06 \pm 0.37$ & $1.33 \pm 0.49$ & $1.39 \pm 0.52$ \\
\hline
\end{tabular}

${ }^{*} p<.05$

Figure 6. Multiple Object Tracking Scores in all three sessions

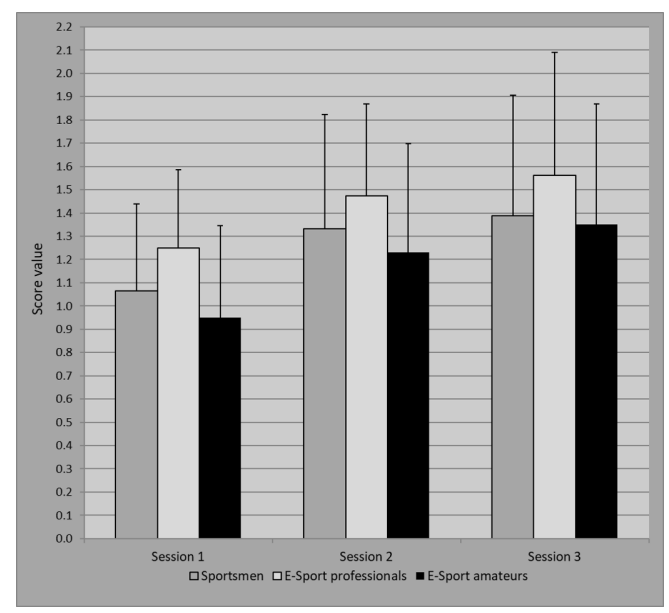

No associations between scores of the other two sessions and weekly playtime or between overall esport participation (in years) and scores of any session were found. No associations between scores of any sessions and weekly sport activity or overall sport participation were found.

\section{DISCUSSION}

The present study evaluated and compared the essential ability of MOT in three groups: professional esport players, amateur esport players and traditional sportsmen. Only tendentious results could be obtained in this comparison. Professional players outperformed amateur players in the first of

Figure 7. Correlation of esport playtime and score values of session one

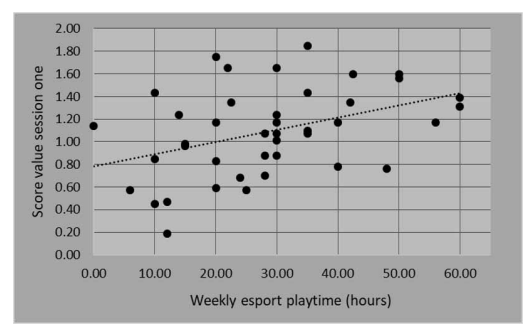


three sessions. Related to this finding, correlation analysis revealed a positive association between weekly esport playtime and score values in session one. No further significant group differences or associations were found.

The first hypothesis can be partly supported, professional players did outperform amateur players, but only in the first session. Professional players might adapt more quickly to the MOT paradigm and therefore initially perform better.

There was support for the second hypothesis, no significant difference between professional players and traditional sportsmen was observed in any of the three sessions. Improvements in attentional and visuo-spatial cognition of professional esport players might be comparable in magnitude to those gained by traditional sportsmen.

There was no support found for the third hypothesis, MOT performance of traditional sportsmen and amateur players did not differ statistically. Playing esport titles on a lower competitive (amateur) level possibly still induces some improvements of MOT performance.

\section{Differences Between Esport Professionals and Amateurs}

In the first session, professional players showed better MOT performance than amateur players. This is somewhat in line with multiple studies that showed how expert video gamers were able to track objects at greater speeds compared to non-experts (Boot et al., 2008; Green \& Bavelier, 2003; Trick et al., 2005). It is important to note that, in those studies, expert video gamers were categorized by the amount of time they played video games (e.g., for multiple years, several times a week for at least one hour), but not by their level of competition, which has also been criticized in the literature (Dale et al., 2020). They might have been experts by former definitions, even if they did not compete in tournaments at all. To our knowledge, the present study is one of the first to compare actual professional players with amateur players. All esport players in the present study would have been classified as experts by the definition in former studies (Boot et al., 2008; Green \& Bavelier, 2003; Trick et al., 2005), that was based solely on playtime. It is conceivable that the entirety of esport players in the present study (professional players and amateur players) would have also outperformed the non-experts of the mentioned earlier studies.

It is already known that general playing of (action-) video games can have a positive effect on tasks like object tracking, that involve attention and visuo-spatial cognition (Hubert-Wallander et al., 2011; Milani et al., 2019; Spence \& Feng, 2010; Trisolini et al., 2018). This finding might be further differentiated by the present study because of the positive correlation between weekly esport play time and first session MOT performance found in the esport cohort. Professional players played more hours per week and outperformed amateur players in session one, but not session two and three. One possible explanation is a higher entry performance or quicker familiarization with the tracking task of players with a very high esport playtime. This effect possibly leveled off during sessions two and three. Additionally, sessions two and three were performed in the 3D version, which might be unfamiliar to all three groups because esport titles usually played by participants are not played in 3D with stereoscopic glasses. This might limit the benefits granted by high esport playtime and negate group differences from session one in later sessions.

\section{No Differences Between Sportsmen and Esport Players}

MOT performance differences between the esport player groups and traditional sportsmen were not statistically significant. The mean score value of the traditional sportsmen group was between professional players and amateur players. Traditional sportsmen in the present study practised their respective sport much shorter than esport players played video games (about three times less often than amateur players and about four and a half times less often than professional players). This might be another indication that both esport as well as traditional sport influence the basic ability of MOT (or underlying skills) and that sportsmen acquire a comparable level by far less time-consuming practice. Unfortunately, our data do not tell which kind of traditional sport impacts MOT performance to a 
greater extent. This is because participants were recruited independently from their sporting focus and we found no associations between specific sporting activities and MOT performance, due to the limited number of participants in each activity. The data also does not reveal an association between traditional sports activity time and MOT performance.

\section{Differences Between Study Participants and General Population}

Scores of the samples are higher than the scores of the normal population without esport or traditional sport context that are documented in the literature (Faubert, 2013; Qiu et al., 2018). This could be due to either:

- Both traditional sport and esport activity and expertise positively influencing MOT performance, which is in line with the literature (Alves et al., 2013; Bejianki et al., 2014; Ding et al., 2018; Fleddermann et al., 2019; Qiu et al., 2018; Trick et al., 2005; Voss et al., 2009), or

- The participants in this study inherently had higher levels of MOT ability than does the general population. These higher MOT ability levels perhaps led these individuals to participate in either esports or traditional sports, due to psychological gratification from inherently superior performance.

The second assumption is fundamentally oriented and difficult to prove directly. It is possible that certain individuals have developed better cognitive abilities for a variety of reasons. Genetical disposition, cognitive training in other fields such as music or fine motor tasks could all lead to MOT abilities above average. This over average abilities could lead to early success in the field of esport or traditional sport, which is known to be a factor for continuous participation (Hollings et al., 2014).

The first assumption on the other hand is broadly described in the literature. Voss et al. (2009) found in their review that expert athletes performed better in tasks that required processing speed and visual attention. This is confirmed by Alves et al. (2013) that found marginally higher performance in comparable measures for elite volleyball players than in amateurs. Qiu et al. (2018) acquired similar results. They analyzed the MOT performance of elite, mediocre and non-athletes in traditional sports and found comparable results to the present study. Elite athletes even outperformed both other groups, whereas in our study professional esport players only slightly outperformed amateurs. Fleddermann et al. (2019) could moreover show a better training effect on cognitive processes of a MOT-task compared to regular ball training. The higher performance level in perceptual-cognitive tasks of experts compared to amateurs was also confirmed for esport. Bejianki et al. (2014) found better perceptional abilities in videogame players compared to a control group and Ding et al. (2018) even more precisely reported higher MOT performance of expert players compared to controls. This is again in line with the assumption of the bullet point. Furthermore, the literature also reflects that MOT can be used to differentiate elite traditional sportsmen from amateur and non-sportive populations (Faubert, 2013; Faubert \& Sidebottom 2012; Qiu et al., 2018), and indicates a positive association to NBA players' performance (Mangine et al., 2014).

\section{MOT as a Possible Chance for Diversified Training}

From the players' and sportsmen's point of view, it is interesting to speculate about realizable benefits of different training methods. It seems as if both esport and sport activity positively influences MOT performance and that a better MOT ability is associated with the professionalism of esport play. Therefore, the adaptation of existing training habits to improve individual's performance provides a promising opportunity. Existing studies show that MOT training improves underlying perceptional abilities and, as a consequence, sports performance (Junye et al., 2015). One study (Romeas et al., 2016) for example found improvement in in-game parameters and therefore proved a transfer effect, in this case, for decision-making and passing accuracy in basketball. Other findings show an increase in MOT ability, but found no transferable effects (Moen et al., 2018) or documented improved basic 
mental skills such as sustained attention or processing speed, but also found no transfer to specific sport related parameters such as jumping performance (Fleddermann et al., 2019). Despite the ambiguous results, it might in summary be of great benefit for both esport players and traditional sportsmen to improve their MOT ability with varying training methods and profit from possible basic ability improvements and transfer effects.

\section{LIMITATIONS}

There are several factors that limit the findings of the study including the sample size, the diverse esport titles played by participants and the diverse fields of sports the sportsmen were recruited from. Also, study design aspects such as daytime of testing, the original purpose of the testing software and the low number of sessions performed must be respected when interpreting the results.

The study compared 19 professional esport players, 22 amateur esport players and 18 traditional sportsmen, which is the lower limit to perform reliable analysis between three groups. The sample size however is bigger than in most comparable studies (Bejianki et al., 2014; Boot et al., 2008; Ding et al., 2018; Hubert-Wallander et al., 2011) as stated in the study design section. The fact that our outcomes are not clearer seems to be due to the actually non-existent or very small differences between the participant groups.

Additionally, the groups of esport-players were not recruited solely from one main played title and the sportsmen were not recruited with the focus on specific fields of sport. This limits the significance of correlation calculations, but can be attributed to practical reasons. It is difficult to attain professional players in general and we decided to open up the field of played titles in order to achieve at least an acceptable overall sample size. As a consequence of the professional players playing several different esport-titles, we also opened up the criteria for amateur players and traditional sportsmen.

As in most studies, participants could freely choose the daytime to participate in the experiment, which leads to possible differences in performance based on personal peaks or lows. This is hardly controllable but is a problem of nearly every comparable study. Also, the freely chooseable breaks in between sessions might influence participants performance. Not granting breaks on the other hand would influence the results even more, because we could not guarantee that participants are as recovered as possible at the beginning of each session.

Another limitation concerns the use of the testing software to compare initial performance. The software is mainly designed as a training tool. It is also used for diagnosis, but on a much rarer basis. The low number of sessions (3) and the different graphical presentation (2D and 3D) make it additionally hard to compare broad data. Maybe the results would become clearer when executing a much higher number of sessions and concentrating on only one graphical presentation. This again is limited by participants availability, especially when testing professional players.

To minimize further impacts on the results, all outcomes were controlled for age and gender which did not result in any change.

\section{CONCLUSION}

The present study is new in that it is the first to compare the MOT performance of three relevant groups: professional esport players, amateur esport players and traditional sportsmen. To our knowledge, no studies that assess the performance of professional players and compare it to other player groups have been published yet. Professional players outperformed amateur players in the first session, which was the only group difference found. Positive association of esport playtime and MOT performance as well as elevated MOT-scores of the entire study cohort compared to normal population indicate a connection of esport and sport activity and tracking performance. As stated in the discussion, other research has collected evidence that advanced esport players as well as expert traditional athletes show higher performance levels in MOT paradigms. This is possibly because of well-trained underlying 
cognitive processes that result in better results for visuo-spatial, visual memory, multifocal and divided attentional tasks. Our results point in the same direction, because a high engagement time in esports seems to correlate with better MOT performance. Additionally, general engagement in either esport or traditional sport seemed to elevate the ability of MOT in the study cohort above the normal population. This connection might be also used to positively influence training habits of both esport players and sportsmen. The findings that a general engagement in esport and traditional sport seems to elevate MOT performance and that both sports training and cognitive training can improve MOT performance offer new training possibilities. Traditional sportsmen could integrate cognitive MOT training at a computer, esport players could engage in traditional sport activities that foster the MOT ability and people that did not engage in either activity might benefit largely from both possibilities.

Future research should focus on eradicating the present limitations such as sample size and diversity of genres the players and sportsmen were recruited from. Larger groups of professional players, amateur players and traditional sportsmen only from one field of esport or sport should be compared in more sessions of MOT, possibly over a larger time interval. This would also give more insight into the learning curves of individuals. It would also be of use to include non-athlete-nonesport participants to examine and emphasize possible benefits of both esport and sport on MOT performance.

\section{FUNDING}

This research was supported by the BGF-institute (= Institut für Betriebliche Gesundheitsförderung; limited liability company) of the AOK Rhineland/Hamburg.

\section{ACKNOWLEDGMENT}

We wish to thank Marius Loewe and Markus Soffner for their assistance in data collection and data analyses and all participants for their contribution. We also want to thank the AOK Rhineland/ Hamburg and the BGF-institute (limited liability company) for their support. 


\section{REFERENCES}

Abbott, A., \& Collins, D. (2004). Eliminating the dichotomy between theory and practice in talent identification and development: Considering the role of psychology. Journal of Sports Sciences, 22(5), 395-408. doi:10.108 0/02640410410001675324 PMID:15160593

Alves, H., Voss, M., Boot, W. R., Deslandes, A., Cossich, V., Inacio Salles, J., \& Kramer, A. F. (2013). Perceptualcognitive expertise in elite volleyball players. Frontiers in Psychology, 4, 36. doi:10.3389/fpsyg.2013.00036 PMID:23471100

Bányai, F., Griffiths, M. D., Király, O., \& Demetrovics, Z. (2019). The psychology of esports: A systematic literature review. Journal of Gambling Studies, 35(2), 351-365. doi:10.1007/s10899-018-9763-1 PMID:29508260

Bavelier, D., Green, C. S., Han, D. H., Renshaw, P. F., Merzenich, M. M., \& Gentile, D. A. (2011). Brains on video games. Nature reviews. Neuroscience, 12(12), 763-768. . 10.1038/nrn3135

Bejjanki, V. R., Zhang, R., Li, R., Pouget, A., Green, C. S., Lu, Z. L., \& Bavelier, D. (2014). Action video game play facilitates the development of better perceptual templates. Proceedings of the National Academy of Sciences of the United States of America, 111(47), 16961-16966. doi:10.1073/pnas.1417056111 PMID:25385590

Blizzard Entertainment. (2010). StarCraft II. Blizzard Entertainment. https://starcraft2.com

Boot, W. R., Kramer, A. F., Simons, D. J., Fabiani, M., \& Gratton, G. (2008). The effects of video game playing on attention, memory, and executive control. Acta Psychologica, 129(3), 387-398. doi:10.1016/j.actpsy.2008.09.005 PMID: 18929349

Cavanagh, P., \& Alvarez, G. A. (2005). Tracking multiple targets with multifocal attention. Trends in Cognitive Sciences, 9(7), 349-354. doi:10.1016/j.tics.2005.05.009 PMID:15953754

Cavanagh, P., Battelli, L., \& Holcombe, A. O. (2014). Dynamic attention. In The Oxford handbook of attention (pp. 503-521). Oxford University Press.

Dale, G., Joessel, A., Bavelier, D., \& Green, C. S. (2020). A new look at the cognitive neuroscience of video game play. Annals of the New York Academy of Sciences, 1464(1), 192-203. doi:10.1111/nyas.14295 PMID:31943260

Ding, Y., Hu, X., Li, J., Ye, J., Wang, F., \& Zhang, D. (2018). What Makes a Champion: The Behavioral and Neural Correlates of Expertise in Multiplayer Online Battle Arena Games. International Journal of HumanComputer Interaction, 34(8), 682-694. doi:10.1080/10447318.2018.1461761

Vancouver, E. A. (2018). FIFA 19. Electronic Arts. https://www.ea.com/de-de/games/fifa/fifa-19

Tiburon, E. A. (2019). Madden NFL 2019. EA Sports. https://www.ea.com/de-de/games/madden-nfl/maddennfl-19

Ensemble Studious. (2005) Age of Empires III. Microsoft Game Studios. https://www.ageofempires.com

Faubert, J., \& Sidebottom, L. (2012). Perceptual-cognitive training of athletes. Journal of Clinical Sport Psychology, 6(1), 85-102. doi:10.1123/jcsp.6.1.85

Faubert, J. (2013). Professional athletes have extraordinary skills for rapidly learning complex and neutral dynamic visual scenes. Scientific Reports, 3(1), 1154. doi:10.1038/srep01154 PMID:23378899

Fleddermann, M. T., Heppe, H., \& Zentgraf, K. (2019). Off-Court Generic Perceptual-Cognitive Training in Elite Volleyball Athletes: Task-Specific Effects and Levels of Transfer. Frontiers in Psychology, 10, 1599. doi:10.3389/fpsyg.2019.01599 PMID:31396123

García-Lanzo, S., \& Chamarro, A. (2018). Basic psychological needs, passion and motivations in amateur and semi-professional eSports players. Aloma: revista de psicologia, ciències de l'educació i de l'esport Blanquerna, 36(2), 59-68.

Green, C. S., \& Bavelier, D. (2003). Action video game modifies visual selective attention. Nature, 423(6939), 534-537. doi:10.1038/nature01647 PMID:12774121

Happonen, A., \& Minashkina, D. (2019). Professionalism in eSport: Benefits in skills and health \& possible downsides. LUT Scientific and Expertise Publications. 
Himmelstein, D., Liu, Y., \& Shapiro, J. L. (2017). An exploration of mental skills among competitive league of legend players. International Journal of Gaming and Computer-Mediated Simulations, 9(2), 1-21. doi:10.4018/ IJGCMS.2017040101

Hollings, S. C., Mallett, C. J., \& Hume, P. A. (2014). The transition from elite junior track-and-field athlete to successful senior athlete: Why some do, why others don't. International Journal of Sports Science \& Coaching, 9(3), 457-471. doi:10.1260/1747-9541.9.3.457

Hubert-Wallander, B., Green, C. S., Sugarman, M., \& Bavelier, D. (2011). Changes in search rate but not in the dynamics of exogenous attention in action videogame players. Attention, Perception \& Psychophysics, 73(8), 2399-2412. doi:10.3758/s13414-011-0194-7 PMID:21901575

Infinity Ward. (2019). Call of Duty: Modern Warfare. Activision. https://www.callofduty.com

Junye, L. Q., Blázquez, A. P., Fortó, J. S. I., \& Torrad, G. C. (2015). Entrenamiento perceptivocognitivo con el Neurotracker 3D-MOT para potenciar el rendimiento en tres modalidades deportivas. Apunts Educación Física y Deportes, (119), 97-108.

Kowal, M., Toth, A. J., Exton, C., \& Campbell, M. J. (2018). Different cognitive abilities displayed by action video gamers and non-gamers. Computers in Human Behavior, 88, 255-262. doi:10.1016/j.chb.2018.07.010

Levitt, H. C. C. H. (1971). Transformed up-down methods in psychoacoustics. The Journal of the Acoustical Society of America, 49(2B), 467-477. doi:10.1121/1.1912375 PMID:5541744

Lipovaya, V., Lima, Y., Grillo, P., Barbosa, C. E., de Souza, J. M., \& Duarte, F. J. D. C. M. (2018). Coordination, communication, and competition in eSports: A comparative analysis of teams in two action games. In Proceedings of 16th European Conference on Computer-Supported Cooperative Work-Exploratory Papers. European Society for Socially Embedded Technologies (EUSSET).

Mangine, G. T., Hoffman, J. R., Wells, A. J., Gonzalez, A. M., Rogowski, J. P., Townsend, J. R., \& Fragala, M. S. et al. (2014). Visual tracking speed is related to basketball-specific measures of performance in NBA players. Journal of Strength and Conditioning Research, 28(9), 2406-2414. doi:10.1519/JSC.0000000000000550 PMID:24875429

Memmert, D. (2009). Pay attention! A review of visual attentional expertise in sport. International Review of Sport and Exercise Psychology, 2(2), 119-138. doi:10.1080/17509840802641372

Milani, L., Grumi, S., \& Di Blasio, P. (2019). Positive Effects of Videogame Use on Visuospatial Competencies: The Impact of Visualization Style in Preadolescents and Adolescents. Frontiers in Psychology, $10,1226$. doi:10.3389/fpsyg.2019.01226 PMID:31231275

Moen, F., Hrozanova, M., Stiles, T., \& Travlos, A. K. (2018). The effects of perceptual-cognitive training with Neurotracker on executive brain functions among elite athletes. Cogent Psychology, 5(1), 1544105. doi:10.10 $80 / 23311908.2018 .1544105$

Mouton, J. D., \& Oberle, C. D. (2007). Discrimination Ability of Traditional and Action-Based Measures of Attentional Style in Sports. American Journal of Psychological Research, 3(1), 78-84.

Nadeo. (2013). Trackmania 2: Stadium. Ubisoft. https://store.steampowered.com/app/232910/TrackMania_ Stadium

Newzoo. (2020). Newzoo Global Esports Market Report 2020 - Light Version. https://newzoo.com/insights/ trend-reports/newzoo-global-esports-market-report-2020-light-version/

Nielsen, R. K. L., \& Karhulahti, V. M. (2017). The problematic coexistence of' internet gaming disorder" and esports. In Proceedings of the 12th International Conference on the Foundations of Digital Games (pp. 1-4). doi:10.1145/3102071.3106359

Oksama, L., \& Hyönä, J. (2004). Is multiple object tracking carried out automatically by an early vision mechanism independent of higher-order cognition? An individual difference approach. Visual Cognition, 11(5), 631-671. doi:10.1080/13506280344000473

Pedraza-Ramirez, I., Musculus, L., Raab, M., \& Laborde, S. (2020). Setting the scientific stage for esports psychology: A systematic review. International Review of Sport and Exercise Psychology, 13(1), 1-34. doi:10 $.1080 / 1750984 X .2020 .1723122$ 
PES Productions. (2019). PES $20=$ Pro Evolution Soccer 20. Konami. https://www.konami.com/wepes/2020/ eu/de/ps4

Polyphony Digital. (2017). Gran Turismo Sport. Sony Interactive Entertainment. https://www.gran-turismo. com/de/products/gtsport

PUBG Corporation. (2017). PlayerUnknown's Battlegrounds. PUBG Corporation. https://www.pubg.com

Pylyshyn, Z. W., \& Storm, R. W. (1988). Tracking multiple independent targets: Evidence for a parallel tracking mechanism. Spatial Vision, 3(3), 179-197. doi:10.1163/156856888X00122 PMID:3153671

Qiu, F., Pi, Y., Liu, K., Li, X., Zhang, J., \& Wu, Y. (2018). Influence of sports expertise level on attention in multiple object tracking. PeerJ, 6, e5732. doi:10.7717/peerj.5732 PMID:30280051

Riot Games. (2009). League of Legends. Riot Games. https://na.leagueoflegends.com

Romeas, T., Chaumillon, R., Labbe, D., \& Faubert, J. (2019). Combining 3D-MOT with motor and perceptual decision-making tasks: conception of a life-sized virtual perceptual-cognitive training paradigm. BioRxiv, 511337. 10.1101/511337

Scholl, B. J. (2009). What have we learned about attention from multiple-object tracking (and vice versa)? In D. Dedrick \& L. Trick (Eds.), Computation, cognition, and Pylyshyn (pp. 49-77). Academic Press.

Spence, I., \& Feng, J. (2010). Video games and spatial cognition. Review of General Psychology, 14(2), 92-104. doi:10.1037/a0019491

Supercell. (2016). Clash Royale. Supercell. https://clashroyale.com

Tabachnick, B. G., \& Fidell, L. S. (2001). Using multivariate statistics (Vol. 5). Allyn \& Bacon.

Taylor, P. (2011). eSport and physical activity f. Torkildsen fs sport and leisure management (6th ed.). Oxon: Routledge.

Torner, H. P., Carbonell, X., \& Castejón, M. (2019). A comparative analysis of the processing speed between video game players and non-players. Aloma: Revista de Psicologia, Ciències de l'Educació i de l'Esport, 37(1). http://orcid.org/0000-0002-9714-1568

Trick, L. M., Jaspers-Fayer, F., \& Sethi, N. (2005). Multiple-object tracking in children: The "Catch the Spies" task. Cognitive Development, 20(3), 373-387. doi:10.1016/j.cogdev.2005.05.009

Trisolini, D. C., Petilli, M. A., \& Daini, R. (2018). Is action video gaming related to sustained attention of adolescents? Quarterly Journal of Experimental Psychology, 71(5), 1033-1039. doi:10.1080/17470218.2017. 1310912 PMID:28335681

Turn 10 Studios. (2011). Forza Motorsport 4. Microsoft Studios. https://forzamotorsport.net/en-US/games/FM4

Valve Corporation. (2013). Defence of the Ancients 2. Valve Corporation. https://www.dota2.com

Valve Corporation. (2012). Counter-Strike: Global Offensive. Valve Cooperation. https://store.steampowered. com/app/730/CounterStrike_Global_Offensive)

Voss, M. W., Kramer, A. F., Basak, C., Prakash, R. S., \& Roberts, B. (2009). Are expert athletes "expert" in the cognitive laboratory? A meta-analytic review of basic attention and perception and sport expertise. Applied Cognitive Psychology, 24, 812-826. doi:10.1002/acp.1588

Williams, A. M., Hodges, N. J., North, J. S., \& Barton, G. (2006). Perceiving patterns of play in dynamic sport tasks: Investigating the essential information underlying skilled performance. Perception, 35(3), 317-332. doi:10.1068/p5310 PMID:16619949 
Konstantin Wechsler $(P h D)$ is a staff member and research associate in the eSports project at the German Sport University Cologne. His main tasks include the management of eSport performance tests and the design of cognitive testing for eSports players.

Peter Bickmann is a staff member and research associate in the eSports project at the German Sport University Cologne. He records and analyzes eye-tracking data of differently skilled eSports players and investigates new approaches for the usage of eye-tracking as a training tool in eSports.

Kevin Rudolf $(P h D)$ is a staff member and research associate in the eSports project at the German Sport University Cologne. His lines of action are the planning and evaluation of eSport survey studies.

Chuck Tholl is a staff member and research associate in the eSports project and doctoral candidate at the German Sport University Cologne. He functions as an instructor for eSports performance tests and adapts classical therapy concepts for eSports.

Ingo Froböse $(P h D)$ is head of the Institute of Movement Therapy and Movement-oriented Prevention and Rehabilitation at the German Sport University Cologne. Professor Froböse navigates the eSports project at the University. As a lateral thinker in science, he founded the eSports project.

Christopher Grieben $(P h D)$ is a research associate in the Institute of Health Promotion and Clinical Movement Science at the German Sport University Cologne. He focuses on research and teaching with regard to the impact of physical activity and sport on health all the way to questions of health communication, ergonomics and product testing. He also chairs the eSports working group at the German Sport University Cologne. 